Rot at i onal f I uct uat i on of nol ecul es in quant um cl usters. II. Nol ecul ar rot at i on and superfl ui di ty in OCS doped hel i um 4 cl ust er s

\begin{tabular}{|l|l|}
\hline 著者 & M ur a Shi ni chi \\
\hline 著者別表示 & 三浦 伸一 \\
\hline $\begin{array}{l}\text { j our nal or } \\
\text { publ i cat i on titl e }\end{array}$ & Journal of Chem cal Physi cs \\
\hline vol une & 126 \\
\hline page range & $114309-1$ - 114309- 7 \\
\hline year & 2007- 01-01 \\
\hline URL & ht t p: //doi . or g/10. 24517/00010275 \\
\hline
\end{tabular}




\title{
Rotational fluctuation of molecules in quantum clusters. II. Molecular rotation and superfluidity in OCS-doped helium-4 clusters
}

\author{
Shinichi Miura ${ }^{a}$ \\ Institute for Molecular Science, 38 Myodaiji, Okazaki 444-8585, Japan
}

(Received 15 December 2006; accepted 9 February 2007; published online 20 March 2007)

\begin{abstract}
In this paper, quantum fluctuations of a carbonyl sulfide molecule in helium- 4 clusters are studied as a function of cluster size $N$ in a small-to-large size regime $(2 \leqslant N \leqslant 64)$. The molecular rotation of the dopant shows nonmonotonic size dependence in the range of $10 \leqslant N \leqslant 20$, reflecting the density distribution of the helium atoms around the molecule. The size dependence on the rotational constant shows a plateau for $N \geqslant 20$, which is larger than the experimental nanodroplet value. Superfluid response of the doped cluster is found to show remarkable anisotropy especially for $N$ $\leqslant 20$. The superfluid fraction regarding the axis perpendicular to the molecular axis shows a steep increase at $N=10$, giving the significant enhancement of the rotational fluctuation of the molecule. On the other hand, the superfluid fraction regarding the axis parallel to the molecular axis reaches 0.9 at $N=5$, arising from the bosonic exchange cycles of the helium atoms around the molecular axis. The anisotropy in the superfluid response is found to be the direct consequence of the configurations of the bosonic exchange cycles. (C) 2007 American Institute of Physics.
\end{abstract}

[DOI: $10.1063 / 1.2713397]$

\section{INTRODUCTION}

In recent years, superfluid helium nanodroplets have attracted great interests in the field of the low temperature chemistry and physics. ${ }^{1-4}$ Quantum fluctuation of the medium has been found to give dramatic impact on chemical processes in the nanodroplet. An impressive example is provided by rotational dynamics of dopant molecules in the nanodroplets. ${ }^{5}$ Spectroscopic measurements on the doped droplets have shown that the solvated molecules rotate freely in an effective manner, owing to the superfluidity of the nanodroplets. Conversely, this indicates that the dopant spectroscopy in the helium nanodroplets offers a unique opportunity to probe the properties of quantum fluids in the finite size. In our companion paper (paper I), ${ }^{6}$ we have presented a path integral hybrid Monte Carlo method for rotating molecules in quantum fluids. In the present paper (paper II), we study the molecular quantum fluctuations of the dopant in helium- 4 clusters and the superfluidity of the doped clusters using the path integral hybrid Monte Carlo method.

Various dopants have been studied both experimentally ${ }^{1,2,5-10}$ and theoretically. ${ }^{3,4,11-20}$ Among them, a carbonyl sulfide (OCS) molecule is one of the most widely studied dopants in the helium nanodroplets. Recently, Tang et ${ }^{7}{ }^{7}$ have determined the vibrational and rotational spectra of the $\mathrm{OCS}(\mathrm{He})_{N}$ clusters in a small-to-medium size regime, $N$ $=2-8$. They found that an effective rotational constant $B_{\text {eff }}$, which is inversely proportional to an effective moment of inertia of the solvated molecule, decreases monotonically as $N$ increases. Importantly, the rotational constant for $N=5$ was

\footnotetext{
${ }^{a)}$ Permanent address: Division of Mathematical and Physical Sciences, Graduate School of Natural Science and Technology, Kanazawa University, Kakuma, Kanazawa 920-1192, Japan. Electronic mail: miura@cphys.s.kanazawa-u.ac.jp
}

found to be almost the same value for the nanodroplet and then overshoot the nanodroplet limit. This indicates the presence of a turnover in the size dependence on $B_{\text {eff }}$ in larger cluster size regime, possibly being related to the onset of the superfluidity in the helium clusters.

From a theoretical view point, the size dependence on the rotational fluctuation of the OCS molecule has been studied by ground-state quantum Monte Carlo (QMC) techniques for the clusters in the small-to-medium size regime. ${ }^{14,15}$ The calculations predicted a minimum in the $N$-dependent rotational constant at $N=8-9$ (Ref. 15) or $N=6$ (Ref. 14); the small discrepancy between the calculations presumably arises from the difference of the He-OCS interaction adopted. Then, the constant increase of $B_{\text {eff }}$ was found up to $N=20$. At a finite temperature corresponding to the experimental condition, a path integral Monte Carlo (PIMC) technique has been applied to a fixed classical OCS molecule dissolved in the helium clusters. ${ }^{3,12}$ Recently, we have developed a path integral hybrid Monte Carlo (PIHMC) method to handle the rotational motion of the dopant molecules quantum mechanically. ${ }^{20}$ Then, the PIHMC was applied to a OCS-doped helium- 4 cluster $\mathrm{OCS}\left({ }^{4} \mathrm{He}\right)_{64}$ to demonstrate the impact of the Bose statistics on the rotational correlation in the solvated molecule. Independently, Zillich et al. ${ }^{19}$ have developed a PIMC method to treat rotating molecules in superfluids and applied the method to the OCS-doped helium clusters. In this paper, the size dependence on the rotational fluctuation of the OCS molecule is systematically studied by the PIHMC method and connected with the superfluidity of the doped clusters in the small-to-large size regime $(N$ $=2-64$ ).

This paper is organized as follows. The method is described in Sec. II. Computational details are given in Sec. III. 
Calculated results on the doped helium-4 cluster OCS $\left({ }^{4} \mathrm{He}\right)_{N}$ are presented in Sec. IV. Summary and discussion are given in Sec. V.

\section{METHOD}

In this section, we summarize the path integral hybrid Monte Carlo method for a rotating molecule in superfluids. Thorough account on the method is described in the paper I. ${ }^{6}$ The doped cluster consists of $N$ helium- 4 atoms obeying the Bose-Einstein statistics and a OCS molecule modeled as a rigid rotor. The partition function of the system $Z$ at an inverse temperature $\beta=1 / k_{B} T$ is written in discretized path integral as ${ }^{21,22}$

$$
\begin{aligned}
Z= & \frac{1}{N !} \sum_{\mathcal{P}} \int \cdots \int \prod_{s=1}^{M} d \mathbf{R}^{(s)} d \mathbf{\Omega}^{(s)} \\
& \times \prod_{s=1}^{M} \rho\left(\mathbf{R}^{(s)}, \boldsymbol{\Omega}^{(s)}, \mathbf{R}^{(s+1)}, \mathbf{\Omega}^{(s+1)} ; \Delta \tau\right),
\end{aligned}
$$

where $\Delta \tau=\beta / M$ is the imaginary time step and $\rho(\Delta \tau)$ is the high temperature density matrix of the system. Here, $\mathbf{R}^{(s)}$ denotes the $3(N+1)$-dimensional position vector including the molecule's center-of-mass position, and $\boldsymbol{\Omega}^{(s)}$ represents the molecule's orientation in the laboratory frame. The superscript $s$ runs from 1 to $M$, labeling the corresponding imaginary time slice. The permutation $\mathcal{P}$ is included in the boundary condition of the path as $\mathbf{R}^{(M+1)}=\mathcal{P} \mathbf{R}^{(1)}$. As described in the paper $I^{6}{ }^{6}$ the high temperature density matrix is approximated based on a hybrid use of the pair density matrix approximation ${ }^{21}$ and the standard factorization technique is accurate up to $\mathcal{O}\left(\Delta \tau^{4}\right)$ (Refs. 22 and 23). Then, the rotational density matrix for a free rotor at the inverse temperature $\Delta \tau, \rho^{\mathrm{rot}}\left(\boldsymbol{\Omega}, \boldsymbol{\Omega}^{\prime} ; \Delta \tau\right)$, which appears in the approximated expression in the high temperature density matrix, is converted into an effective potential of quantum rotation $u^{\text {rot }}$ by the following relation: $\rho^{\mathrm{rot}}(\Delta \tau) \equiv e^{-\Delta \tau u^{\mathrm{rot}}\left(\mathbf{\Omega}, \mathbf{\Omega}^{\prime}\right)}$. This effective potential accounts for the rotational kinetic energy in a form of the potential function. Using the above expressions, the partition function can be cast in the following form:

$$
\begin{aligned}
Z= & \frac{1}{N !} \sum_{\mathcal{P}} \prod_{i=1}^{N+1}\left(\frac{m_{i} M}{2 \pi \beta \hbar^{2}}\right)^{3 M / 2} \int \cdots \int\left\{\prod_{s=1}^{M} d \mathbf{R}^{(s)} d \mathbf{\Omega}^{(s)}\right\} \\
& \times e^{-S(\{\mathbf{R}\},\{\mathbf{\Omega}\}, \mathcal{P})},
\end{aligned}
$$

where $m_{i}$ is the physical mass of the $i$ th particle. The imaginary time action $S$ is written by

$$
\begin{aligned}
S= & \sum_{s=1}^{M} \sum_{i=1}^{N+1} \frac{m_{i} M}{2 \beta \hbar^{2}}\left(\mathbf{r}_{i}^{(s)}-\mathbf{r}_{i}^{(s+1)}\right)^{2}+\Delta \tau \sum_{s=1}^{M} u^{\mathrm{rot}}\left(\mathbf{\Omega}^{(s)}, \mathbf{\Omega}^{(s+1)}\right) \\
& +\Delta \tau \sum_{s=1}^{M}\left\{U_{\mathrm{He}-\mathrm{He}}\left(\mathbf{R}^{(s)}, \mathbf{R}^{(s+1)}\right)+V_{\mathrm{He}-\mathrm{OCS}}\left(\mathbf{R}^{(s)}, \boldsymbol{\Omega}^{(s)}\right)\right. \\
& \left.+V_{\text {corr }}\left(\mathbf{R}^{(s)}, \mathbf{\Omega}^{(s)}\right)\right\}
\end{aligned}
$$

where $\mathbf{r}_{i}^{(s)}$ denotes the position of the $i$ th particle at the imaginary time slice $s$. The harmonic interaction in the first term arises from the translational kinetic energy and the sec- ond from the rotational kinetic energy. The $\mathrm{He}-\mathrm{He}$ interaction is described by an effective potential $U_{\mathrm{He}-\mathrm{He}}$ defined using the pair density matrix of helium atoms ${ }^{24,25}$ and the $\mathrm{He}-\mathrm{OCS}$ interaction by $V_{\mathrm{He}-\mathrm{OCS}}$ and $V_{\text {corr }}$; the former comes from the direct $\mathrm{He}-\mathrm{OCS}$ interaction and the latter from the fourth order correction by Marx and Müser ${ }^{22}$ and Takahashi and Imada. ${ }^{23}$ When we omit the term $V_{\text {corr }}$, we recover the hybrid expression of the pair density matrix and the primitive approximations, which was used in the path integral Monte Carlo calculations of helium-4 clusters doped with fixed classical molecules. ${ }^{3,12}$ The expressions of $V_{\mathrm{He}-\mathrm{OCS}}$ and $V_{\text {corr }}$ are explicitly described in Paper I. ${ }^{6}$ Then, the path variables $\left(\mathbf{R}^{(1)}, \ldots, \mathbf{R}^{(M)}\right)$ and $\left(\boldsymbol{\Omega}^{(1)}, \ldots, \boldsymbol{\Omega}^{(M)}\right)$ are sampled using the hybrid Monte Carlo move which is realized by introducing the fictitious linear and angular momenta. This enables us to simultaneously update the path variables in an efficient manner. On the other hand, the permutation $\mathcal{P}$ and the associated path variables are updated based on a multilevel Metropolis move $^{21}$ combined with a configurational-bias technique. ${ }^{26}$

\section{COMPUTATIONAL DETAILS}

The calculated system consists of $N$ helium- 4 atoms and a OCS molecule. The cluster size studied ranges from smallto-large cluster regime: $N=2-12,15,20,40$, and 64 . Temperature of the system was controlled to be $0.37 \mathrm{~K}$. The number of discretization needed to path integral expression was chosen to be $M=216$ corresponding to $1 / \Delta \tau=80 \mathrm{~K}$. The rotational constant of the OCS molecule was taken from a gas-phase experimental value ${ }^{27} B_{\mathrm{OCS}}=0.20286 \mathrm{~cm}^{-1}$ to construct the effective potential of quantum rotation on a fine grid. The Aziz potential ${ }^{28}$ was used as a pairwise interaction between two helium atoms. The pair density matrix of helium-4 atoms was evaluated numerically by a matrix squaring technique, ${ }^{21}$ which defines the effective interaction $U_{\mathrm{He}-\mathrm{He}}$ among the helium- 4 atoms. ${ }^{24,25}$ The morphed potential of Howson and Hutson ${ }^{29}$ was adopted for the He-OCS interaction. Path integral hybrid Monte Carlo calculations were performed for the system obeying the Bose-Einstein statistics. For comparison, the systems obeying the MaxwellBoltzmann statistics were also examined.

\section{RESULTS}

\section{A. Molecular fluctuations}

We first show the size dependence on translational and rotational fluctuations of the OCS molecule. The translational fluctuation is described by the following mean square correlation function of the center of mass of the OCS molecule:

$$
R^{2}(\tau)=\left\langle\left|\mathbf{r}_{N+1}(\tau)-\mathbf{r}_{N+1}(0)\right|^{2}\right\rangle .
$$

The correlation function $R^{2}(\tau)$ is a periodic function in the imaginary time with a period $\beta$. The translational fluctuation is well characterized by $R^{2}(\tau)$ at $\tau=\beta / 2$ which reflects the spatial dispersion of the center of mass of the molecule. Regarding the full time dependence, see Fig. 4 in Paper I. ${ }^{6}$ The $R^{2}(\beta / 2)$ for a free OCS molecule can be written analytically by $^{30}$ 


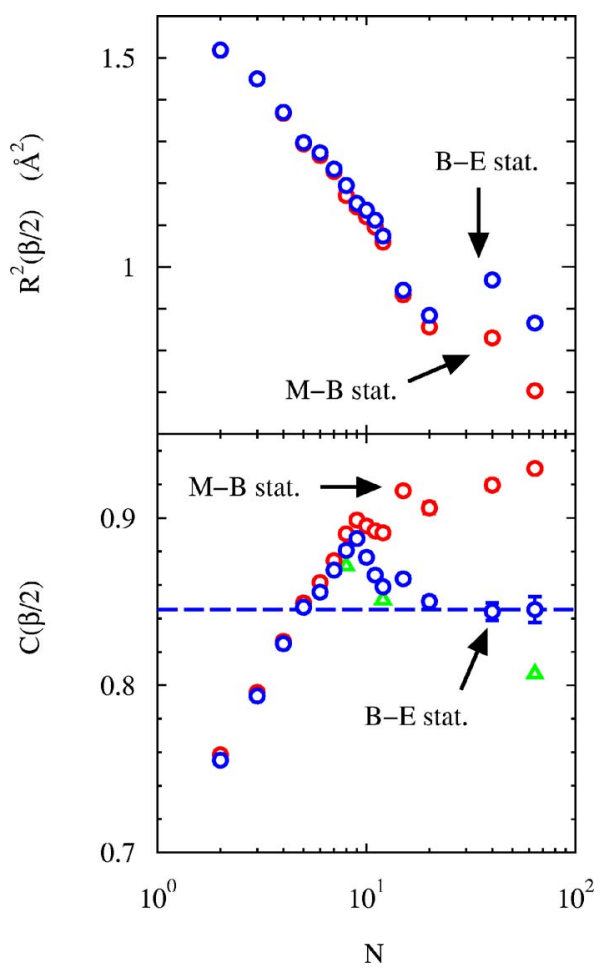

FIG. 1. (Color online) Upper panel: The imaginary time mean square correlation function of the OCS center-of-mass position at the imaginary time $\beta / 2, R^{2}(\beta / 2)$, as a function of the cluster size $N$. Blue open circles represent the results of the Bose clusters and red open circles the results of the Boltzmann clusters. Lower panel: The imaginary time orientational correlation function of the OCS at the imaginary time $\beta / 2$ as a function of the cluster size $N$. Blue open circles are for the Bose clusters and red open circles for the Boltzmann clusters. Dashed line indicates the $C(\beta / 2)$ value for $N=64$. Green open triangles represent the $C(\beta / 2)$ values by path integral Monte Carlo calculations of the Bose clusters doped with the OCS molecule (Ref. 19). The error bar is expressed at 95\% confidence level and is smaller than the size of the corresponding data symbol when it is not shown.

$$
R^{2}(\beta / 2)=\frac{3 \beta \hbar^{2}}{4 m_{\mathrm{OCS}}}
$$

where $m_{\mathrm{OCS}}$ denotes the physical mass of the OCS molecule. For the free OCS molecule, the $R^{2}(\beta / 2)$ was evaluated to be $1.64 \AA^{2}$. For the solvated molecule in the helium- 4 clusters, the $R^{2}(\beta / 2)$ as a function of the cluster size $N$ is presented in Fig. 1. In both Boltzmann and Bose clusters, the spatial dispersion becomes suppressed with increasing $N$. This is the result of the interaction of the OCS with the surrounding helium atoms. The effect of the Bose statistics is found to be not so large for the translational fluctuation, although the $R^{2}(\beta / 2)$ value for the Bose cluster is systematically larger than the Boltzmann counterpart for $N>5$. The larger spatial fluctuation found in the Bose clusters can be ascribed to the slightly broader density distribution of the helium atoms around the molecule by taking into account the Bose statistics, which gives a weaker effect of the spatial confinement.

The rotational fluctuation of the molecule is examined by the following orientational correlation function in the imaginary time:

$$
C(\tau)=\langle\mathbf{e}(\tau) \mathbf{e}(0)\rangle,
$$

where $\mathbf{e}(\tau)$ is a unit vector of the molecular axis at the imaginary time $\tau$. As in the translational fluctuation, the rotational fluctuation of the molecule is characterized by $C(\tau)$ at $\tau$ $=\beta / 2$. Since $\mathbf{e}(\tau) \mathbf{e}(0)=\cos \gamma(\tau)$, where $\gamma(\tau)$ is an angle between $\mathbf{e}(\tau)$ and $\mathbf{e}(0)$, the smaller $C(\beta / 2)$ value indicates larger orientational fluctuation. The $C(\beta / 2)$ can be written for a free OCS in an analytical form ${ }^{31}$

$$
C(\beta / 2)=\frac{\sum_{J>0} 2 J e^{-\beta B_{\mathrm{OCS}} J^{2}}}{Z},
$$

where $Z=\Sigma_{J \geqslant 0}(2 J+1) e^{-\beta B_{\mathrm{OCS}} J(J+1)}$. The value of $C(\beta / 2)$ for the free OCS was evaluated to be 0.65 . Figure 1 shows the size dependent $C(\beta / 2)$ of the OCS molecule in the Boltzmann and Bose clusters. The Boltzmann $C(\beta / 2)$ monotonically increases with $N(\leqslant 9)$ and reaches a maximum at $N$ $=9$. Second maximum in the size dependence is found at $N$ $=15$. For $N \geqslant 20$, the $C(\beta / 2)$ again increases monotonically up to $N=64$. On the other hand, the Bose $C(\beta / 2)$ first increases with $N(\leqslant 5)$ where the effect of the Bose statistics is negligible. Then, the Bose $C(\beta / 2)$ starts to deviate from the Boltzmann $C(\beta / 2)$ with $N$, indicating that the rotational fluctuation becomes larger than that of the OCS molecule doped in the Boltzmann cluster. In the size range of $N \geqslant 10$, the effect of the Bose statistics becomes remarkable; the magnitude of the $C(\beta / 2)$ is much reduced compared with the Boltzmann counterpart, although the presence of the two peaks is a common feature to the Bose and Boltzmann clusters. For $N \geqslant 20$, the $C(\beta / 2)$ has a plateau in the size dependence, which shows clear contrast with the Boltzmann clusters where $C(\beta / 2)$ still increases over $N=20$. Recently, Zillich et al. ${ }^{19}$ have performed path integral Monte Carlo calculations for $\operatorname{OCS}\left({ }^{4} \mathrm{He}\right)_{N}$ clusters for $N=8,12$, and 64 . Although size dependence on $C(\beta / 2)$ by their calculations shows similar trend with our calculations for the selected cluster sizes, the rotational fluctuation of the OCS molecule for $N=64$ by their calculation is much larger than that by our calculation. Although this is partly ascribed to the difference of the He-OCS interaction adopted, systematic analysis about the size dependence on the rotational fluctuation using their He-OCS interaction is needed to fully understand the observed discrepancy.

Here, we estimate the effective rotational constant $B_{\text {eff }}$ of the solvated molecule using the above $C(\beta / 2)$ value. The estimation procedure is the same as that presented in paper $\mathrm{I},{ }^{6}$ using the calculated $C(\beta / 2)$ value and the analytical expression of $C(\beta / 2)$, Eq. (7). Figure 2 shows the estimated effective rotational constant $B_{\text {eff }}$ as a function of $N$, together with the experimental data ${ }^{7,32}$ and the reported ground-state quantum Monte Carlo values. ${ }^{15}$ The effective rotational constant by the present study decreases with increasing $N$ up to 9 and then shows a turnover. We find a second (weak) minimum at $N=15$ corresponding to the second maximum in the size dependence on $C(\beta / 2)$. The effective rotational constant for $N \geqslant 20$ shows a plateau that is larger than the experimental nanodroplet value. Our estimated $B_{\text {eff }}$ values are in good agreement with the experimental $B_{\text {eff }}$ where the experimental data are available. Also good agreement of the present results with the QMC values is found up to $N=20$. The size dependence on $B_{\text {eff }}$ will be discussed in Sec. V. 


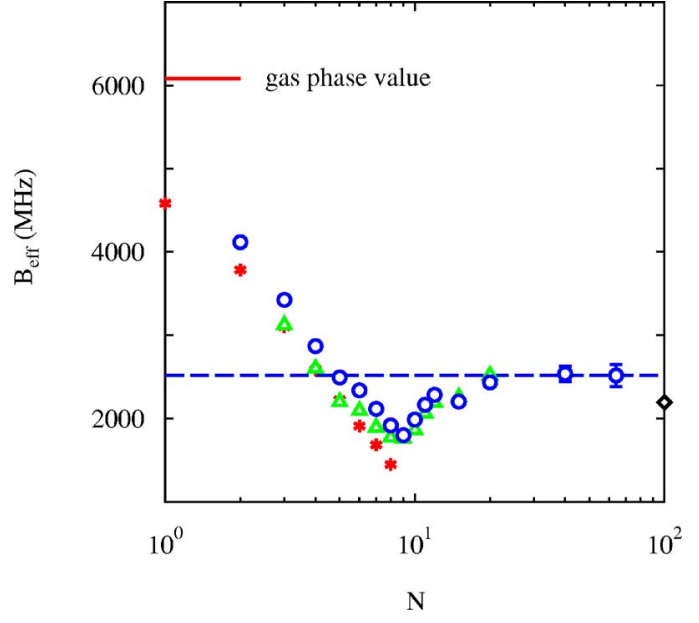

FIG. 2. (Color online) The effective rotational constant $B_{\text {eff }}$ as a function of the cluster size $N$. Blue open circles represent the estimated values for Bose clusters and green open triangles $B_{\text {eff }}$ by the ground-state quantum Monte Carlo calculations (Ref. 15). Star symbols show the experimental $B_{\text {eff }}$ reported in Ref. 7. Open diamond indicates the experimental nanodroplet value (Ref. 32). The dashed line indicates the $B_{\text {eff }}$ value of $N=64$. The error bar is estimated so as to be consistent with the statistical error of $C(\beta / 2)$ in Fig. 1.

\section{B. Solvation structure}

We next show the local density distribution of the helium atoms measured from the center of mass of the OCS molecule $\rho(\mathbf{r})$, characterizing the solvation structure developed around the OCS molecule. For a linear molecule, we can write $\rho(\mathbf{r})=\rho(z, r)$ in terms of cylindrical coordinates. The results on selected clusters are presented in Fig. 3. At $N=5$, all the helium atoms are found to be localized around the $\mathrm{C}$ atom where the He-OCS interaction is a minimum, forming a doughnut-type structure of the density around the molecular axis. Then, the helium atoms start to populate around other potential minima located near the $\mathrm{O}$ and $\mathrm{S}$ atoms for larger clusters. At $N=6$ and 7, the region near the $\mathrm{O}$ atom is first occupied by the helium atoms. Following this, at $N=8$ and 9 , another minimum around $\mathrm{S}$ atom starts to be filled by the helium atoms. At $N=10$, the helium atoms are found to populate in the whole region in the vicinity of the molecule. For $N>10$, density augmentation is observed in the intermediate region between the $\mathrm{S}$ and $\mathrm{C}$ atoms in the solvation shell, making a new peak of the density distribution. For $N$ $>15$, the addition of the helium atoms induces the reconfiguration of the solvation shell; the peak near the $\mathrm{S}$ atom becomes lowered and shifted to the direction of the $\mathrm{C}$ atom along the solvation shell. At $N=20$, the first solvation shell is nearly completed. For $N>20$, the second solvation shell starts to be filled by the helium. In this case, the structure of the first solvation shell itself is unchanged by adding the helium atoms, although the density increase in the first solvation shell is observed for $N \leqslant 64$. Here, we decompose the density profile on the basis of the length of the exchange cycles to extract the effect of the Bose statistics. In Fig. 4, we present the density profile of the helium atoms involved in long exchange cycles with the length $P \geqslant 6$. In the case of $N=5$, the helium density in the five-body exchange cycles is presented. At $N=5$, the five-body exchange cycles are observed to a large extent; in this case, the contributions from the two- to four-body cycles are negligible. These exchange cycles are located around the molecular axis in the doughnut structure. For $6 \leqslant N \leqslant 9$, the exchange cycles start to be observed in the region over the $\mathrm{C}$ and $\mathrm{O}$ atoms. Interestingly, for $N=8$ and 9 , the component from the long exchange cycles is negligible in the region over the $\mathrm{C}$ and $\mathrm{S}$ atoms, although the helium atoms are populated in that region. This is due to the absence of the helium density in the intermediate region between the $\mathrm{C}$ and $\mathrm{S}$ atoms, prohibiting the bosonic exchange of the helium atoms located in the difference potential minima. At $N=10$, the bosonic exchange between the $\mathrm{C}$ and $\mathrm{S}$ regions starts to be visible, indicating that the exchange cycles of the helium atoms are present to wrap

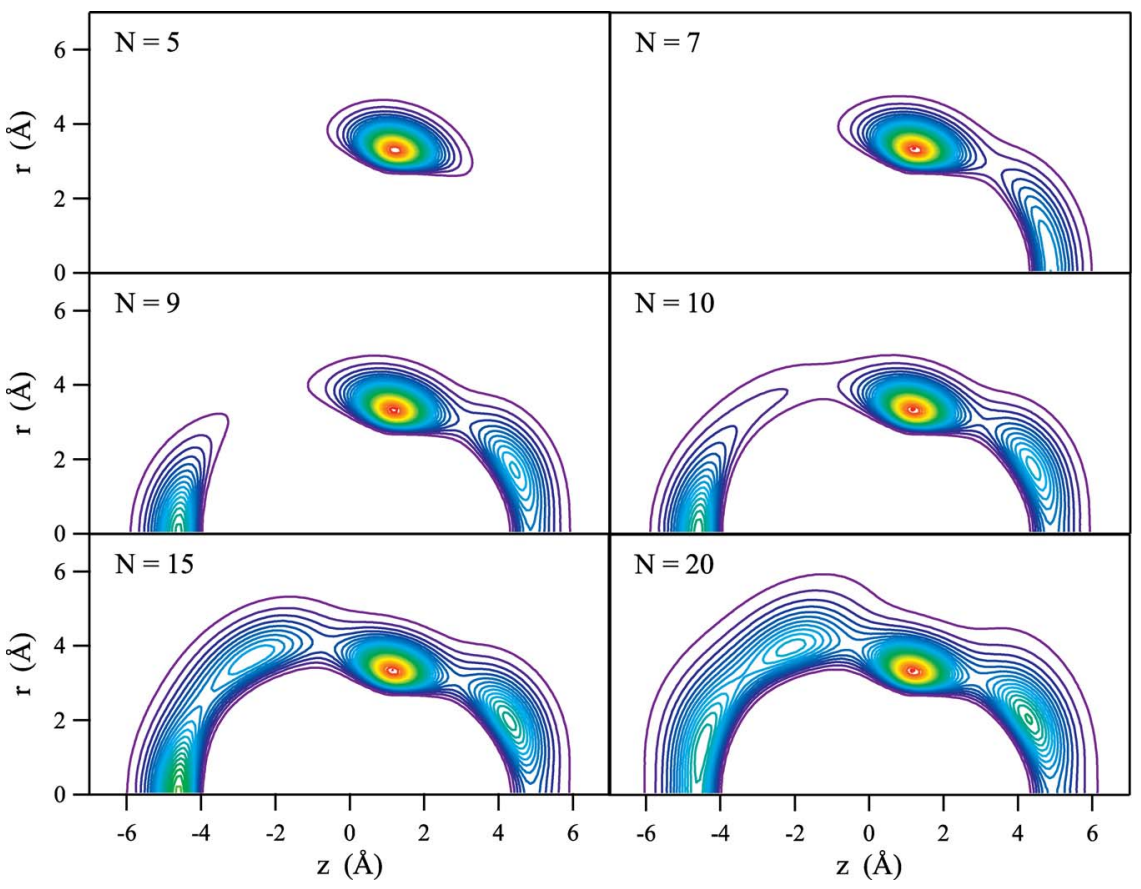

FIG. 3. (Color online) Contour plot of helium density distribution $\rho(z, r)$ for selected Bose clusters. The OCS molecule is located on the $z$ axis with the center of mass at $z=0$; the oxygen, carbon, and sulfur atoms are aligned from the right to the left on the $z$ axis. All densities are in $\AA^{-3}$. Color scale goes from 0 to $0.17 \AA^{-3}$. 


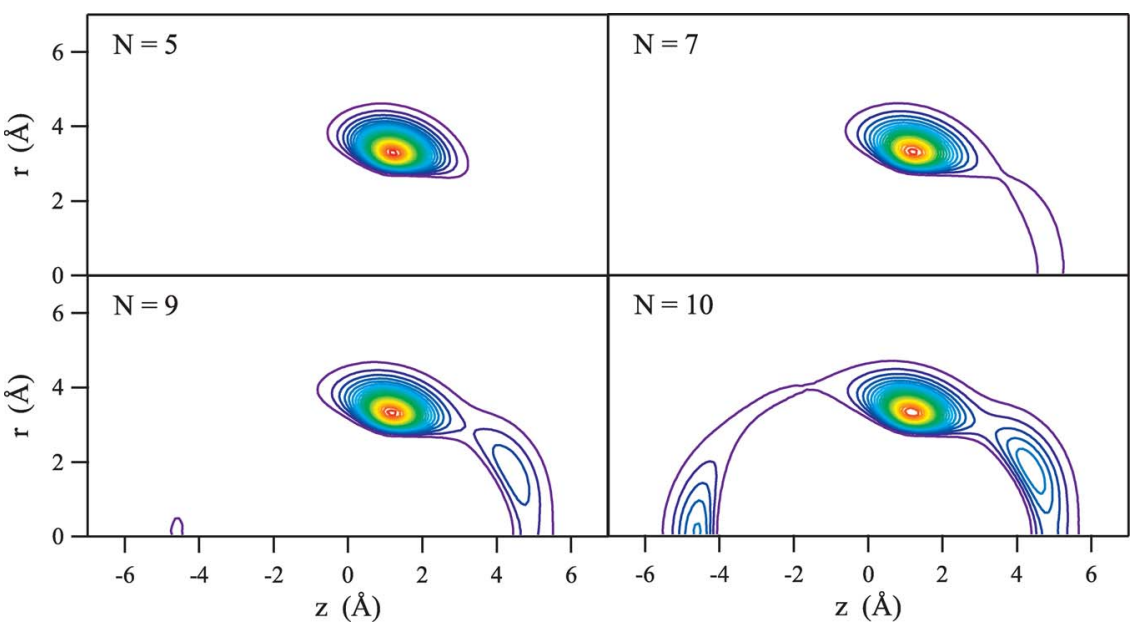

FIG. 4. (Color online) Contour plot of density distribution of the helium atoms participating in the exchange cycles with the length $P \geqslant 6 ; \rho^{(6)}(z, r)$ is presented for selected Bose clusters. In the case of $N=5, \rho^{(5)}(z, r)$ which is the density of the helium atoms participating in fivebody exchange cycles is presented. The OCS molecule is located on the $z$ axis with the center of mass at $z=0$; the oxygen, carbon, and sulfur atoms are aligned from the right to the left on the $z$ axis. All densities are in $\AA^{-3}$. Color scale goes from 0 to $0.055 \AA^{-3}$. the OCS molecule in the plane including the molecular axis. With increasing $N$, the contribution from the long exchange cycles becomes larger. For $N \geqslant 20$, the long exchange cycles give the dominant contribution in the density distribution.

\section{Superfluidity}

Finally, we discuss the superfluidity of the doped clusters. The superfluid fraction $\rho^{s} / \rho$ about an axis $\mathbf{n}$ is calculated by ${ }^{21,33}$

$$
\left.\frac{\rho^{s}}{\rho}\right|_{\mathbf{n}}=\frac{2 m_{\mathrm{He}}}{\beta \lambda_{\mathrm{He}} I_{c}}\left\langle(\mathbf{A n})^{2}\right\rangle,
$$

where $m_{\mathrm{He}}$ is the physical mass of the helium atom, $I_{c}$ is the classical moment of inertia of the cluster about the axis $\mathbf{n}$, and $\lambda_{\mathrm{He}}=\hbar^{2} / 2 m_{\mathrm{He}}$. The area vector $\mathbf{A}$ is defined by

$$
\mathbf{A}=\frac{1}{2} \sum_{s=1}^{M} \sum_{i=1}^{N} \mathbf{r}_{i}^{(s)} \times \mathbf{r}_{i}^{(s+1)}
$$

In the present study, the axis considered is defined in such a way that the laboratory frame coincides with the body-fixed frame of the molecule at $\tau=0$. In order to examine the anisotropic response of the system, the superfluid fraction is calculated about the axis perpendicular to and parallel to the molecular axis, denoted, respectively, as $\rho_{\perp}^{s} / \rho$ and $\rho_{\|}^{s} / \rho$. Figure 5 shows $\rho_{\perp}^{s} / \rho$ and $\rho_{\|}^{s} / \rho$ as a function of $N$. As seen in the figure, a remarkable anisotropy in the superfluid response is observed especially for $N \leqslant 20$. Regarding the perpendicular component associated with $C(\tau)$, the superfluid fraction gradually increases with $N$. A steep increase is found around $N=10$ where the turnover of the $C(\beta / 2)$ is found. This increase in the superfluid fraction is attributed to the long exchange cycles wrapping the OCS molecule discussed above, which give a large component of the area $\mathbf{A}$ about the perpendicular axis. This onset of the superfluidity regarding the perpendicular axis causes the enhancement of the rotational fluctuation of the molecule. At $N=20$ where the first solvation shell is almost completed, $\rho_{\perp}^{s} / \rho$ amounts to more than 0.8 . On the other hand, the parallel component of the superfluid fraction $\rho_{\|}^{s} / \rho$ shows quite different $N$ dependence compared with $\rho_{\perp}^{s} / \rho$. The parallel superfluid fraction quickly increases with $N$ and reaches the value 0.9 at $N=5$. This large superfluid fraction arises from the five-body exchange cycles formed around the molecular axis. Since the area vector is almost parallel to the molecular axis by this type of configurations, the perpendicular superfluid fraction remains to be a small value. For larger clusters, $\rho_{\|}^{s} / \rho$ is found to be weakly dependent on $N$. This indicates that the observed anisotropy in the superfluid density directly reflects the configurations of the long exchange cycles. Here, we comment on the finite size effect on the superfluid fraction, which vanishes in the large clusters. This can be estimated from the "superfluid fraction" of the doped Boltzmann clusters. For $N=2$, the superfluid fraction was calculated to be about 0.1 for both the perpendicular and parallel components. Then, the value gradually decreases with increasing $N$, indicating that the above superfluid response is the consequence of the Bose statistics. Note that an another type of the estimator of the superfluid fraction has been used for the helium clusters doped with a rotating molecule. ${ }^{34-36}$ In their calculations, the projected area $\mathbf{A} \cdot \mathbf{n}$ in Eq. (8) is replaced by $\frac{1}{2} \sum_{s} \sum_{i} \mathbf{n}^{(s)}\left[\mathbf{r}_{i}^{(s)}\right.$ $\left.\times \mathbf{r}_{i}^{(s+1)}\right]$, where $\mathbf{n}^{(s)}$ is an axis rotating with the body-fixed

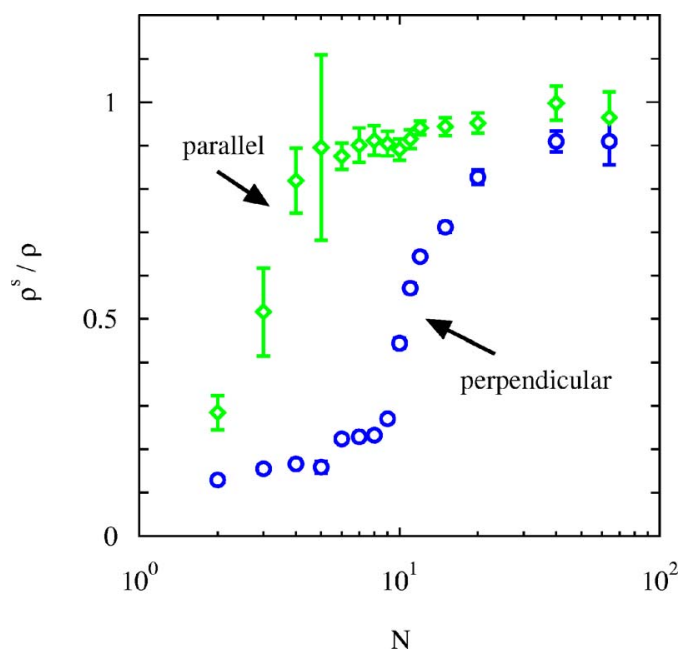

FIG. 5. (Color online) Superfluid fraction of the cluster is presented as a function of the cluster size $N$. Blue open circles represent the component of the superfluid fraction perpendicular to the molecular axis of the OCS and green open diamonds parallel to the molecular axis. The error bar is expressed at $95 \%$ confidence level and is smaller than the size of the corresponding data symbol when it is not shown. 


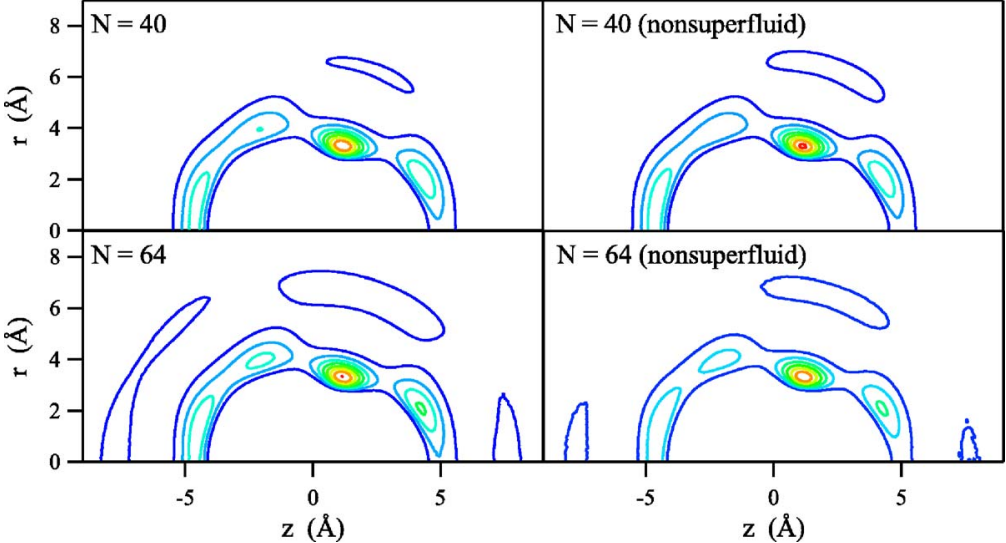

FIG. 6. (Color online) Contour plot of helium density $\rho(z, r)$ (left column) and nonsuperfluid density $\rho^{n}(z, r)$ (right column) for the Bose clusters, $N=40$ and 64. The OCS molecule is located on the $z$ axis with the center of mass at $z=0$; the oxygen, carbon, and sulfur atoms are aligned from the right to the left on the $z$ axis. All densities are in $\AA^{-3}$. Color scale goes from 0 to $0.17 \AA^{-3}$ for $\rho(z, r)$ and from 0 to $0.041 \AA^{-3}$ for $\rho^{n}(z, r)$. frame of the molecule in the imaginary time. While the area vector, Eq. (9), does not depend on the position of the origin, the above projected path area on the time-dependent axis has the origin dependence. In the present study, the area vector projected on an axis defined in the body-fixed frame at the imaginary time $\tau=0$, which is free from the origin dependence, is used to estimate the anisotropic superfluid fraction.

\section{SUMMARY AND DISCUSSION}

In this paper, we have studied the molecular fluctuations of the dopant and the superfluidity of the helium-4 clusters of the size $2 \leqslant N \leqslant 64$ using the path integral hybrid Monte Carlo method. While the translational fluctuation is not affected by the Bose statistics (especially for $N \leqslant 20$ ), the rotational fluctuation shows rich size dependence. Remarkable enhancement of the molecular rotation in the Bose cluster is found for $N \geqslant 10$, being connected to the onset of the superfluidity regarding the axis perpendicular to the molecular axis. We also find that the rotational fluctuation and the associated effective rotational constant show nonmonotonic size dependence in the medium size regime of $10 \leqslant N \leqslant 20$. Although the effect of the Bose statistics is significant in this size regime, the similar trend in the rotational fluctuation is observed even in the doped Boltzmann clusters, implying turnovers themselves reflect the density distribution around the molecule. For $20 \leqslant N \leqslant 64$, the rotational constant of the molecule in the Bose cluster shows a plateau which is larger than the experimental nanodroplet $B_{\text {eff }}$.

Very recently, McKellar et al. ${ }^{10}$ have reported the experimental effective rotational constant of the OCS doped in the helium cluster up to $N=72$. They found that the size dependent $B_{\text {eff }}$ has two minima in $N \leqslant 20$. This finding is in good accordance with the theoretical $B_{\text {eff }}$ presented in this paper. More importantly, they found that the experimental $B_{\text {eff }}$ shows unexpected oscillatory behavior in a medium-to-large size regime, $N>20$, which does not reach the nanodroplet limit yet. In the present study, these broad oscillations were not well resolved, since only two sizes, $N=40$ and 64 , were examined in this size regime. However, the trend in the experimental value was reproduced by our calculations for the selected clusters. McKellar et al. interpreted the oscillations as a manifestation of the Aufbau of a nonclassical helium solvation shell structure. In order to gain an insight on the solvation shell from the present calculations, we show the helium density distribution and a nonsuperfluid density distribution $\rho^{n}(z, r)$ for $N=40$ and 64 in Fig. 6. Here, the nonsuperfluid density is defined using the helium atoms involved in short exchange cycles up to the length $P=5$. It is found that the total helium density in the first solvation shell still augments with increasing $N$ at least up to $N=64$, even after the first solvation shell is almost completed around $N=20$. The second solvation shell is found to be built up in an anisotropic manner, which reflects the He-OCS interaction. Regarding the nonsuperfluid density, the overall structure of the solvation shell is found to be similar with that of the total density, although the magnitude is much smaller than the total density. Interestingly, the primary peak of $\rho^{n}(z, r)$ in the first solvation shell becomes lowered with increasing $N$, as opposed to the trend of the total density. This comes from the development of the long exchange cycles covering the whole cluster, which promotes the exchange of the helium atoms between the first and second solvation shells. These competing factors might yield the broad oscillations in the rotational constant in the medium-to-large size regime. To reach the definitive conclusion, however, we need more precise calculations on the size dependence to resolve the oscillatory structures observed in the experiment. Finally, bridging the gap between the large clusters and the nanodroplets is the remaining issue. Larger cluster calculations about $N \sim 10^{3}$ are fruitful to understand the behaviors in the unresolved size regime. To this end, further methodological improvements on the path integral hybrid Monte Carlo method are required. These issues will be addressed in the future study.

\section{ACKNOWLEDGMENTS}

The author thanks the Research Center for Computational Science, National Institutes of Natural Sciences for the use of supercomputers. This work was supported by the Next Generation Super Computing Project, Nanoscience Program, MEXT, Japan.

\footnotetext{
${ }^{1}$ J. P. Toennies and D. F. Vilesov, Annu. Rev. Phys. Chem. 49, 1 (1998), and references therein.

${ }^{2}$ J. P. Toennies and D. F. Vilesov, Angew. Chem., Int. Ed. 43, 2622 (2004), and references therein.

${ }^{3}$ Y. Kwon, P. Huang, M. V. Patel, D. Blume, and K. B. Whaley, J. Chem. Phys. 113, 6469 (2000), and references therein.

${ }^{4}$ M. Barranco, R. Guardiola, S. Hernández, R. Mayol, J. Navarro, and M. Pi, J. Low Temp. Phys. 142, 1 (2006), and references therein.

${ }^{5}$ S. Grebenev, J. P. Toennies, and A. F. Vilesov, Science 279, 2083 (1998).
} 
${ }^{6}$ S. Miura, J. Chem. Phys. 126, (2007), previous paper.

${ }^{7}$ J. Tang, Y. Xu, A. R. W. McKellar, and W. Jäger, Science 297, 2030 (2002).

${ }^{8}$ Y. Xu, W. Jäger, J. Tang, and A. R. W. McKellar, Phys. Rev. Lett. 91, 163401 (2003).

${ }^{9}$ J. Tang, A. R. W. McKellar, F. Mezzacapo, and S. Moroni, Phys. Rev. Lett. 92, 145503 (2004).

${ }^{10}$ A. R. W. McKellar, Y. Xu, and W. Jäger, Phys. Rev. Lett. 97, 183401 (2006).

${ }^{11}$ Y. Kwon and K. B. Whaley, Phys. Rev. Lett. 83, 4108 (1999).

${ }^{12}$ Y. Kwon and K. B. Whaley, J. Chem. Phys. 115, 10146 (2001).

${ }^{13}$ E. W. Draeger and D. M. Ceperley, Phys. Rev. Lett. 90, 065301 (2003).

${ }^{14}$ F. Paesani, A. Viel, F. A. Gianturco, and K. B. Whaley, Phys. Rev. Lett. 90, 073401 (2003).

${ }^{15}$ S. Moroni, A. Sarsa, S. Fantoni, K. E. Schmidt, and S. Baroni, Phys. Rev. Lett. 90, 143401 (2003).

${ }^{16}$ S. Moroni, N. Brinov, and P.-N. Roy, J. Chem. Phys. 121, 3577 (2004).

${ }^{17}$ F. Paesani and K. B. Whaley, J. Chem. Phys. 121, 4180 (2004).

${ }^{18}$ S. Paolini, S. Fantoni, S. Moroni, and S. Baroni, J. Chem. Phys. 123, 114306 (2005).

${ }^{19}$ R. E. Zillich, F. Paesani, Y. Kwon, and K. B. Whaley, J. Chem. Phys. 123, 114301 (2005)

${ }^{20}$ S. Miura, J. Phys.: Condens. Matter 17, S3259 (2005).

${ }^{21}$ D. M. Ceperley, Rev. Mod. Phys. 67, 279 (1995), and references therein.
${ }^{22}$ D. Marx and M. H. Müser, J. Phys.: Condens. Matter 11, R117 (1999), and references therein.

${ }^{23}$ M. Takahashi and M. Imada, J. Phys. Soc. Jpn. 53, 3765 (1984).

${ }^{24}$ S. Miura and S. Okazaki, J. Chem. Phys. 115, 5353 (2001).

${ }^{25}$ S. Miura and J. Tanaka, J. Chem. Phys. 120, 2160 (2004).

${ }^{26}$ D. Frenkel and B. Smit, Understanding Molecular Simulation: From Algorithms to Applications, 2nd ed. (Academic, New York, 2002).

${ }^{27}$ N. Hunt, S. C. Foster, J. W. C. Johns, and A. R. W. McKellar, J. Mol. Spectrosc. 111, 42 (1985).

${ }^{28}$ R. A. Aziz, A. R. Janzen, and M. Moldover, Phys. Rev. Lett. 74, 1586 (1995).

${ }^{29}$ J. M. M. Howson and J. M. Hutson, J. Chem. Phys. 115, 5059 (2001).

${ }^{30}$ A. L. Nichols III, D. Chandler, Y. Singh, and D. M. Richardson, J. Chem. Phys. 81, 5109 (1984).

${ }^{31}$ N. Blinov, X. G. Song, and P.-N. Roy, J. Chem. Phys. 120, 5916 (2004).

${ }^{32}$ S. Grebenev, M. Hartmann, M. Havenith, B. Sartakov, J. P. Toennies, and A. F. Vilesov, J. Chem. Phys. 112, 4485 (2000).

${ }^{33}$ P. Sindzingre, M. L. Klein, and D. M. Ceperley, Phys. Rev. Lett. 63, 1601 (1989)

${ }^{34}$ N. Blinov and P.-N. Roy, J. Low Temp. Phys. 140, 253 (2005).

${ }^{35}$ Y. Xu, N. Blinov, W. Jäger, and P.-N. Roy, J. Chem. Phys. 124, 081101 (2006).

${ }^{36}$ W. Topic, W. Jäger, N. Blinov, P.-N. Roy, M. Botti, and S. Moroni, J. Chem. Phys. 125, 144310 (2006). 